Canadian

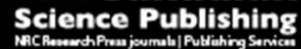

Canadian Journal of Microbiology Revue canadienne de de microbiologie

\title{
Seasonal Variation in an Acid Mine Drainage Microbial Community
}

\begin{tabular}{|r|l|}
\hline Journal: & Canadian Journal of Microbiology \\
\hline Manuscript ID & cjm-2016-0215.R2 \\
\hline Manuscript Type: & Article \\
\hline Date Submitted by the Author: & 09-Sep-2016 \\
\hline Complete List of Authors: & $\begin{array}{l}\text { Auld, Ryan; Laurentian University, Chemistry \& Biochemistry } \\
\text { Mykytczuk, Nadia; Laurentian University, Biology } \\
\text { Leduc, Leo; Laurentian University, Biology } \\
\text { Merritt, Thomas; Laurentian University, Chemistry \& Biochemistry }\end{array}$ \\
\hline Keyword: & $\begin{array}{l}\text { Acid mine drainage, 16S/18S rRNA, temporal variation, microbial } \\
\text { community, community diversity }\end{array}$ \\
\hline
\end{tabular}




\title{
Seasonal Variation in an Acid Mine Drainage Microbial Community
}

\author{
Ryan R. Auld*, Nadia C.S. Mykytczuk $\neq$, Leo G. Leduc ${ }^{\Uparrow}$, Thomas J.S. Merritt* \\ *Department of Chemistry \& Biochemistry, Laurentian University \\ ₹ School of the Environment, Laurentian University \\ I Department of Biology, Laurentian University
}

\section{Corresponding Authors: Thomas Merritt, Department of Chemistry and Biochemistry, Laurentian University, Sudbury, Ontario, Canada, P3E 2C6, Tel: (705) 675-1151, ext 2189, Fax: (705) 675-4844, email: tmerritt@laurentian.ca, Nadia Mykytcuk, School of the Environment, Laurentian University, Sudbury, Ontario, Canada, P3E 2C6 Tel: (705) 675- 1151 Ext 4832 email: $\underline{\text { nx mykytczuk@laurentian.ca }}$}

\begin{abstract}
Environmental oxidation and microbial metabolism drive production of Acid Mine Drainage (AMD). Understanding changes in the microbial community, due to geochemical and seasonal characteristics, is fundamental to AMD monitoring and remediation. We used direct sequencing of the $16 \mathrm{~S}$ and 18S rRNA genes to identify Bacterial, Archaeal, and Eukaryote members of the microbial community at an AMD site in Northern Ontario, Canada, finding a dynamic community varying significantly across winter and summer sampling times. Community composition was correlated with physical and chemical properties including water temperature,
\end{abstract}


$\mathrm{pH}$, conductivity, winter ice thickness, and metal concentrations. Within Bacteria, Acidithiobacillus was the dominant genus during winter (11-57\% of sequences) but Acidiphilium during summer (47-87\%). Within Eukaryotes, Chrysophyceae (1.5-94\%) and Microbotrymycetes $(8-92 \%)$ dominated the winter community and LKM11 (4-62\%) and Chrysophyceae (25-87\%) the summer. The Archaeal domain was less diverse and less variable, with similar summer and winter communities comprised mainly of Thermoplasmata (33-64\%) and Thermoprotei (5-20\%) classes, but also including a large portion of unclassified reads ( $40 \%)$. Overall, the active AMD community, varied significantly between winter and summer, with changing community profiles closely correlated to specific differences in AMD geochemical and physical properties, including $\mathrm{pH}$, water temperature, ice thickness, and sulphate and metal concentrations.

\section{Keywords:}

Acid mine drainage, 16S/18S rRNA, temporal variation, microbial community, community diversity

\section{Introduction}

Acid mine drainage (AMD), acidic water produced from some mining operations, is characterized by low $\mathrm{pH}$ and high metal and mineral concentrations (e.g. iron, nickel, copper, and cadmium), is the largest environmental problem facing the global sulfide mineral mining industry (Egiebor and Oni 2007). It is estimated that in Canada, seven billion tons of mine tailings cover approximately 41,000 hectares of land, with an estimated remediation cost between two and five billion dollars (MEND 1994). AMD production is largely a function of 
microbial oxidation of iron sulfides in the mining tailings. To successfully limit AMD production, and promote remediation of existing sites, we need to control or harness these microbes; understanding the role that the organisms play in driving Fe/S cycling is a large part of this goal. Central to this understanding is describing the diversity of AMD microorganisms and their variation through time and across changes in environment (Hallberg 2010; Volant et al. 2014). Ultimately, a greater understanding of microbe community variation is fundamental to targeted microbe-, site-, and season-specific AMD remediation strategies.

The AMD microbial community varies through time, but the extent of this variation is not well understood. Previous work suggests that AMD communities change with seasonal differences in physical and geochemical properties of the local environment (Edwards et al. 1999; Kay et al. 2013; Leduc et al. 2002; McGinness and Johnson, 1993; Schrenk et al. 1998; Volant et al. 2014), similar to variation seen in other microbial communities (e.g. costal marine waters, Gilbert et al. 2011 or arctic soils, Buckeridge et al. 2013). Members of the genus Acidithiobacillus are often among the most common species inhabiting AMD sites (MendezGarcia et al. 2015), with iron and sulfur oxidizing species in this genus At. ferrooxidans, At. ferridurans, and At. ferrivoran, varying greatly in their abundance across sites and environmental conditions (Liljeqvist et al. 2011; Remonsellez et al. 2009, Tan et al. 2009). In addition, other AMD microorganisms, including Leptospirillum group III, Gallionella ferruginea, and the Archaea Ferroplasma spp., can dominate the community under specific geochemical conditions, (Bond et al. 2000; Hallberg et al. 2006; Huang et al. 2011; Tan et al. 2007).

The use of modern, culture-independent, techniques has allowed the identification of microorganisms previously uncultivated in AMD environments, including A-, E-, G-, and Iplasma Thermoplasmatales (Yelton et al. 2013), ARMAN Archaeal groups (Baker et al.2006), 
and Legionella (Hao et al. 2010; Auld et al. 2013). Continued modern techniques and -omics related research into AMD systems will continue to expand our understanding of site specific, seasonal, and geochemical properties and their relationship to AMD microbial communities. These techniques will also allow better detection of rare AMD organisms, such as Granulicella, Acidocella, and Alicyclobacillus species (e.g. Auld et al. 2013), that despite their low abundance may serve critical niche roles in the AMD environment. For instance, Liljeqvist et al. (2015) recently described cold-adapted Acidithiobacillus sp. and Acidobacteria and Gallionellaceae-like species, driving low temperature AMD processes in the Kristineberg mine in Sweden. It is evident that the cold-active AMD community harbours different bacterial members, many of which do not yet have clearly defined metabolic roles in the environment, and even less known are the Archaeal and Eukaryote members that help shape the low temperature AMD community.

In our study, samples taken during three separate dates during the summer and three separate dates during the winter allowed us to characterize variation in the AMD community during the temperature extremes of Northern Ontario. The active AMD site studied here is part of a long term study ( $>20$ years) in which the drainage pond completely freezes and thaws over the seasons, allowing for potentially unique AMD site and community characteristics. Here we show substantial seasonal variation (variation between 3 summer and 3 winter time points) in the Prokaryote and Eukaryote AMD community composition and species abundance in a nickel mine tailings pond facility in Copper Cliff, ON, Canada using direct sequencing of $16 \mathrm{~S}$ and $18 \mathrm{~S}$ rRNA genes and correlate some of this variation to changes in geochemical and physical AMD properties. 


\section{Methods}

AMD Sample Collection \& Physicochemical Analysis

Acid mine drainage (AMD) water samples were collected from Vale's Copper Cliff Central Tailings Area (located in the City of Greater Sudbury), Ontario (46.47169 $\mathrm{N}$ -

$81.07913^{\circ} \mathrm{E}$ ) in 2012 . Due to the active nature of the mine site, many site details cannot be disclosed, although decades of monitoring records indicate this seepage pond is chemically consistent (1-3g/L total $\mathrm{S}, 3-6 \mathrm{~g} / \mathrm{L}$ total iron, $\mathrm{pH}$ range $2.5-3.5)$ between years and similar to several adjacent ponds draining the area. Water samples were collected on three winter and three summer dates to determine inter-, and intra-, seasonal variation in water chemistry and AMD microbial community composition. Within a season, collections were approximately two weeks apart. The winter dates were: Feb $22^{\text {nd }}$ (hereafter referred to as W1), Mar $1^{\text {st }}(\mathrm{W} 2)$, and Mar $14^{\text {th }}$ (W3) 2012. The summer dates were: July $19^{\text {th }}(\mathrm{S} 1)$, Aug $2^{\text {nd }}(\mathrm{S} 2)$, and Aug $21^{\text {st }}(\mathrm{S} 3) 2012$. All collections were made at approximately 10am EST. To better assess the overall microbial community composition and diversity, water collected from three pond locations (A, B, and CFigure 1) was pooled; locations A and B were several feet off the shoreline and approximately 75 meters apart, while location $\mathrm{C}$ was more central within the pond. Winter samples were taken by drilling holes through surface ice. Summer samples were taken from the shore for the two shallow samples $(\sim 1 \mathrm{~m}$ below surface), and using hip waders and a collection pole for the central sample. In all cases, care was taken not to disturb the sediment during sampling. Due to safety reasons on the active mine site as well as the unevenness of winter ice thickness throughout the pond, access to samples throughout the pond was limited. For each collection time point, three litres of water were aseptically sampled using sterile $1 \mathrm{~L}$ bottles (i.e. nine 1L bottles in total on 
each sampling date). Equal portions from pond locations $\mathrm{A}, \mathrm{B}$, and $\mathrm{C}$ were pooled to give three two litre samples, which were used for cell and DNA extractions (i.e. triplicate water samples). The remaining $1 \mathrm{~L}$ of pooled AMD was used for geochemical analysis.

\section{AMD Geochemistry Analysis}

The average geochemical drainage properties were estimated using the $1 \mathrm{~L}$ pooled water samples from each sampling time. Total metals were estimated using inductively coupled plasma mass spectrometry (ICP-MS) to quantify cations, and ion chromatography to quantify anions; all analyses were conducted by TestMark Laboratory (http://www.testmark.ca). Using separate samples $\mathrm{pH}$, alkalinity, and conductivity were quantified immediately upon return to the lab using Metrohm meters (Herisau, Switzerland). Water and air temperature, ice thickness, and cumulative snow depth were recorded on site for each sampling time. Total dissolved oxygen was not measured during field sampling.

\section{DNA Extraction, PMA Treatment, \& Pyrosequencing}

Microbial cells were filtered from the triplicate, 2L, pooled AMD samples using a $0.2 \mu \mathrm{m}$ membrane filter. One membrane filter was used per 1L of AMD for more efficient cell filtration (i.e. two filters were required per replicate). Cells were collected from the membrane filter by submerging the filters in $7 \mathrm{~mL}$ of sterile AMD and lightly vortexing until the majority of precipitate was seen to be removed from the filters. Propidium monoazide (PMA) was added to each $7 \mathrm{~mL}$ cell sample to a concentration of $50 \mu \mathrm{M}$, followed by 5 min incubation in the dark, and 7 min incubation under a 500 watt light source with constant shaking. PMA treatment crosslinks free DNA from dead cells, but not DNA from active, viable cells; the crosslinking prevents the free DNA from participating in the sequencing reaction, allowing DNA from the active (viable) 
microbial community (which is unmodified by the PMA) to be better sequenced (Nocker et al. 2006). Following PMA treatment, samples were centrifuged for 10 minutes at 5,000 rpm to pellet the cells and the supernatant was discarded. The pellet was re-suspended in $500 \mu \mathrm{L}$ of sterile AMD water and DNA immediately extracted using a MO BIO Power Water DNA isolation Kit, following the manufacturer protocol (MO BIO Laboratories, Inc. California, USA). The extracted DNA was sent to Research and Testing Laboratories (http://researchandtesting.com/) for sequencing on a Roche 454 FLX/FLX + pyrosequencing platform following a modified version of the amplicon pyrosequencing (bTEFAP) process (Dowd et al. 2008). For Bacteria, we targeted the V1-V3 region of the 16S rRNA gene using the 28F (5'-GAGTTTGATCNTGGCTCAG-3'; Paster et al. 2002) and 519R (5'-

GTNTTACNGCGGCKGCTG-3'; Andreotti et al. 2011) universal Bacterial primers. For Archaea, we targeted the V3-V4 regions using the Arch349F (5'-

GYGCASCAGKCGMGAAW-3') and Arch806R (5'- GGACTACHVGGGTWTCTAAT-3'; Caporaso et al. 2011) universal Archaeal primers. For Eukaryotes, we targeted the V1-V3 region of the 18S rRNA gene using the universal Euk7F (5'- AACCTGGTTGATCCTGCCAGT-3'; Medlin et al. 1988) and Euk570R (5'- GCTATTGGAGCTGGAATTAC-3'; Weekers et al. 1994) primers.

\section{Processing of Pyrosequencing Data}

Bacterial and Archaeal 16S rRNA sequences were quality filtered using Qiime 1.5.0 (Caporaso et al. 2010) set to default parameters, removing sequence lengths below 200bp, above $1000 \mathrm{bp}$, or those that did not meet a minimum quality score of 25 . Sequence data was denoised using Qiime default parameters. The complete sets of filtered sequences were classified using the Ribosomal Database Project -II classifier (RDP-II). Classification was performed to the 
class and genus levels using an $80 \%$ threshold (Wang et al. 2007). The Eukaryote 18S rRNA sequences were also processed using Qiime along with quality filtering identical to that of the prokaryote data and Qiime default parameters. Eukaryote sequences were classified to phylum and class level using Qiime, and OTU classification was assigned using the Silva 111 release (Quast et al. 2013), clustering at 97\% sequence identity. OTU's were checked for chimeric sequences using ChimeraSlayer (Haas et al. 2011).

\section{Phylogenetic analyses}

Separate phylogenetic analyses were performed on the dominant taxa $(>1 \%$ of sample sequence reads) from the Bacterial, Eukaryotic, and Archaeal OTU libraries. Representative prokaryotic OTU sequences were selected using the RDP pipeline as follows. Qiime filtered sequencing data was aligned and complete linkage clustering performed using the RDP pipeline with a maximum distance of $15 \%$. Representative sequences were then chosen from the clusters using a maximum distance of $3 \%$, and dominant representatives for each sampling date were used in the phylogenetic analysis along with the two closest nBLAST hits (Altschul et al. 1990). nBLAST searches were performed against cultured organisms first and those sequences that showed no matches were then searched using nBLAST against environmental uncultured microorganisms. Phylogenetic analysis was performed using the Molecular Evolutionary Genetics Analysis software package (MEGA 5; Tamura et al. 2011) using maximum likelihood and the Tamura-Nei model with 1000 bootstrap replicates. A phylogeny of dominant $(>1 \%$ sequence reads) Eukaryotic sequences was constructed using MEGA 5 (Tamura et al. 2011) in similar fashion to the analyses of Bacterial and Archaeal sequences.

\section{Statistical Comparison of Communities}


Alpha (within community) and beta (between community) diversity analysis for both Prokaryote and Eukaryote datasets were performed using Qiime. The Bacterial, Archaeal, and Eukaryote community sequencing datasets were quality filtered and clustered at the $97 \%$ sequence identity within each domain. Representative sequences for all three domains were chosen and aligned using the default PyNAST parameter and representative sequences were aligned using the core Silva 108 aligned set implemented in Qiime. Alignments were filtered using default parameters and phylogenies for statistical analyses were built using fasttree in order to perform beta analyses. Principle coordinate analysis, Unifrac distances, Unifrac significance, and P-tests were performed on rarefied (equal sampling depths) datasets to compare within and between seasons. Chao1 (Chao 1987) and Shannon (Shannon and Weaver 1949) diversity indices were calculated on rarefied data using Qiime to determine the species richness and evenness of distribution.

Statistical Analysis of chemical properties and microbial community profiles

Multivariate correlation analysis (Redundancy Analysis - RDA) was performed with Canoco 4.0 (Ithaca, New York, USA), and RDA triplots were created, to identify possible correlations between physical and chemical AMD properties with variation in the microbial community composition across the sample dates. Additionally, Spearman's rank correlations were calculated with JMP version 10 (SAS - Cary, North Caroline, US) to test possible correlation between taxonomic groups and AMD chemical and physical properties. All multivariate analyses were performed on all genera that were present at more than two seasonal points.

\section{Results}


Environmental Analyses

Geochemical and physical AMD properties were measured for each sampling time to potentially identify temporal trends. The $\mathrm{pH}$, temperature, conductivity, and concentration of several key AMD metals are plotted across the sampling times in Figure 2. Metal concentrations and conductivity decreased over the course of the winter and drainage $\mathrm{pH}$ was found to decrease at the end of winter to 2.94 from that of the first two winter samples of 3.27 and 3.28 respectively. In contrast, metal concentrations were fairly constant during the summer, with only a slight increase through time. The $\mathrm{pH}$ and conductivity remain relatively constant in the summer however, the $\mathrm{pH}$ values were lower in summer (between $2.75-2.8$ ) than in winter. Average yearly, winter, and summer metal concentrations are shown in Table 1 and the complete measured AMD properties for the six sampling dates are shown in Supplemental Table 1.

rRNA Direct Sequencing Analysis

Bacterial 16S, Archaeal 16S, and Eukaryote 18S small subunit rRNA gene sequences were directly sequenced from total, PMA-treated, DNA extracted from the AMD water samples. Over 525,000 trimmed and quality filtered gene sequence reads were analyzed to assess the microbial diversity of the AMD site over the six sampling dates (Supplemental Table 2). Bacteria and Eukaryotes were relatively common at all sampling times; we obtained sequence data for the Bacterial and Eukaryote domains from all six sampling dates (Supplemental Figure 1). In contrast, Archaeans were relatively rare and we were only able to obtain Archaean sequences from the winter samples (935, 1,733, and 19,589 sequences), and the last summer sample (405 sequences). 
Acid Mine Drainage Microbial Community Profile

Bacterial Community

We compared the AMD Bacterial communities between each sampling time using Unifrac's phylogenetic based metric, (Lozupone and Knight 2005) depicted as PCoA plots based on seasonal microbial community change and species abundance (Figure 3A). The composition of the AMD microbial community varied both within and between seasons. The Bacterial PCoA plot illustrates tight seasonal clusters, with tighter clustering in summer than in winter suggesting a more similar, and constant, summer AMD Bacterial community. For each Bacterial seasonal dataset, a Unifrac phylogeny based distance metric was calculated and examined in addition to PCoA clustering trends (Supplemental Figure 2). Unifrac distances confirmed the PCoA analyses with larger variation between than within seasons, with a greater diversity of OTUs identified within the winter samples. Both Unifrac's phylogeny based significance test (indicating variation in clade length) and P-tests (lineage specific variation - variation in community composition) indicated significant differences in the bacterial community between and within seasons $(<0.001)$. The Bacterial kingdom was found to have the greatest biodiversity for both Chao1 (which estimates the number of species present) and Shannon (which estimates both species diversity and evenness of the community population) estimates of species richness. Further, Chao1 and Shannon bacterial indices were consistently higher during the winter (Mean Choa1 $=1,114$; Shannon $=5.55)$ than during the summer $($ Chao1 $=741$; Shannon= 4.02).

Across all samples, three classes of Proteobacteria make up the majority of the Bacterial AMD community, Betaproteobacteria, Gammaproteobacteria, and Alphaproteobacteria, with the relative contribution of each group varying across samples, especially between seasons (Figure 4A). The Bacterial summer community in S1 and S2 is largely composed of 
Alphaproteobacteria (88\% and 85\%) and Betaproteobacteria (9\% and 10\% relative abundance, respectively). The final summer community (S3) was significantly different from those of S1 and S2 (Unifrac significance test $<0.001$ ), with Alphaproteobacteria and Gammaproteobacteria comprising $48 \%$ and $47 \%$ relative abundance. During the winter months, Betaproteobacteria and Gammaproteobacteria dominated the AMD, but follow different abundance trends. Betaproteobacteria decrease throughout winter from $52 \%$ relative abundance in $\mathrm{W} 1$ to $6 \%$ in W3. Conversely, the Gammaproteobacteria initially represented $14 \%$ of the winter classified reads but increase to nearly $62 \%$ in our last winter sample (W3). Also during the winter months, the population of Alphaproteobacteria remain relatively constant at $1-4 \%$ relative abundance. The differences in the dominant bacterial members between the summer and winter seasons are readily apparent in the phylogenetic tree of representative OTUs ( $>1 \%$ sequence reads, Figure 5).

More than $90 \%$ of Bacterial sequences from the summer samples were confidently classified to genus (Figure 4B) and overall, the summer Bacterial diversity was found to be relatively low compared to winter. The summer community was dominated by Acidithiobacillus, ranging from $8 \%$ relative abundance in S1 to over 36\% in S3, and Acidiphilium, ranging from $87 \%$ to $47 \%$ between S1 and S3 sampling dates. Legionella sequences were also found in the samples from all sequencing dates, although in low read numbers except for the final S3 summer sample ( $8 \%$ relative sequence reads).

Classification of winter Bacterial sequences was more challenging than summer classification: only $28 \%$ of $\mathrm{W} 1,47 \%$ of $\mathrm{W} 2$, and $63 \%$ of $\mathrm{W} 3$ could be classified to genus. The classified W1 sequences included, Acidithiobacillus (11\% of total sequences), Herminiimonas (6\%), Flavobacterium (3\%), Hyphomicrobium (2\%) and several other low abundance $(<1 \%)$ genera. While all genera present in the summer were also present in the winter, there were a 
number of genera that were only found in winter, including Flavobacterium, Hyphomicrobium, Sphingomonas, Ignavibacterium, Sulfuricella, and Sulfuritalea (Figure 4B). The largest winter community shift was the increase of Acidithiobacillus from $11 \%$ in W1, to $32 \%$ in W2, and $57 \%$ in W3. The genus Leptospirillum, commonly found in AMD systems (Schrenk et al. 1998; Bond et al. 2000), was also found throughout the year but only in relatively low abundance ranging from a high of $3.5 \%$ in $\mathrm{W} 3$ to less than $1 \%$ in all other, winter and summer, samples.

\section{Archaeal Community}

Across all samples, Archaeal abundance and biodiversity (both classified and unclassified) were low compared to the other two domains (Supplemental Figure 1 \& Figure 6). All classified sequences were from only two classes, Thermoprotei and Thermoplasmata, but a large percentage of sequence data could not be classified (see below). However, even with this relative low abundance and diversity, the seasonal communities are easily resolved in the PCoA plot (Figure 3B). Unifrac weighted and unweighted distances matrices were greater between seasons than within winter (within season numbers for summer could not be calculated with one sample), indicating that the seasonal data were distinct $(<0.001)$, but that there was no significant variation across the winter samples (Supplemental Figure 3). Species and richness estimates for Archaea were the lowest of the three domains with a winter average of 59 species and 3.21 Shannon index, and an increasing trend was found as the winter proceeded.

A large portion of the Archaeal sequences $(26-56 \%)$ could only be classified to the Archaeal domain and could not be confidently classified to phylum (not shown), class (Figure 6A) or genus (Figure 6B). This lack of resolution reflects the continued lack of sequence information from AMD Archaea, and Archaea in general. The sequences that could be classified 
to class suggest the Archaeal community is largely composed of Thermoplasmata and

Thermoprotei with 32 - 60\% and 4 - 20\% relative abundances, respectively.

Thermogymnomonas and Ferroplasma were the only genera confidently identified, and both were found in highest abundance in W3 at 17\% and 12\%, respectively (Figure 6B). The genus Thermoplasma was also identified during the winter but in extremely low percentages $(<0.1 \%$ abundance).

\section{Eukaryote Community}

The Eukaryote microbial community was a measurable part of the AMD microbial community, with lower diversity than Bacteria, but much higher abundance and diversity than Archaea (Supplemental Table 3). The Eukaryotic community composition varied significantly between seasons and tight seasonal clustering is apparent in the PCoA plot (Figure $7 \&$ Figure 3C). Further, there was significant variation in clade lengths (evolutionary difference) and lineage specific variation between (Unweighted Unifrac Significance $<0.001$, and P-Test $<0.001$ ) and within seasons (P-Test $<0.05$ - Supplemental Figure 4). Weighted and unweighted Unifrac significance tests however, indicated no significant differences within season samples. Diversity estimates for Eukaryotes showed less variation between seasons than for Bacteria or Archaea, with an estimated 207 species during the winter, compared to 192 summer species, with Shannon indices of 4.75 and 3.51 , respectively.

We identified three dominant classes within the Eukaryote sequences (Figure 7): Chrysophyceae (1.5-94\%) a large group of algae, Microbotrymycetes (0-92\%) a group of fungi, and LKM11 (0-62\%) a novel fungal clade. Communities in the summer months were comprised of both fungal and algal Eukaryotes, in which Chrysophyceae algae dominated during the early 
summer (87-25\%) and the fungal group LKM11 became more dominant during late summer (4$65 \%$ ). In contrast, the winter communities were comprised largely by algal species, namely Chrysophyceae in the earlier winter samples (W1 and W2; 85, 95\%) and then a shift to Microbotrymycetes in the last winter sample (W3; over 91\%).

\section{Correlations with AMD Environmental Parameters}

As a first step to understanding the forces shaping the microbial community, we compared the observed seasonal patterns in microbial community structure to our environmental parameters using multivariate and redundancy analyses. Four AMD properties (pH, conductivity, temperature and ice formation), and five geochemical AMD properties (iron, copper, sulfate, sulfur, and nickel concentrations) were compared to the abundance of Bacterial genera as well as Archaeal and Eukaryote classes. Redundancy analysis (RDA) triplots show the relationship between the AMD properties and the Bacterial and Eukaryote abundances and community structure (Figure 8). The winter Bacterial community was dominated by Acidithiobacillus that was negatively correlated with conductivity (Spearman's rank correlation coefficient $\rho=-0.89,|\rho|=0.019)$ and sulfate concentration $(\rho=-0.94,|\rho|=0.0048)$ along with Herminiimonas and Flavobacterium abundances that both positively correlated with $\mathrm{pH}$ and ice thickness $(\rho>0.88,|\rho|=0.021)$ and negatively correlated with water temperature $(\rho<-0.88$, $|\rho|=0.021)$. Leptospirillum abundance only significantly correlated with ice thickness $(\rho=0.83$, $|\rho|=0.042$ ), likely reflecting increased sequence abundance during the winter months. The summer community was dominated by Acidiphilium abundance that positively correlated with drainage temperature $(\rho=0.77,|\rho|=0.072)$ and negatively with $\mathrm{pH}(\rho=-0.77,|\rho|=0.072)$. 
Similarly, the Eukaryotic groups also had complex patterns of associations with the AMD environmental parameters. The dominant winter Eukaryote community including Spirotrichea, Saccharomycetes and Agaricomycetes were all positively related to ice thickness $(\rho=0.93,|\rho|=$ 0.008, 0.83, $|\rho|=0.042$ and $0.89|\rho|=0.019$ respectively). Leotiomycetes and Dothideomycetes both negatively correlated with sulfur concentrations $(\rho=-0.88,|\rho|=0.018 \&-0.83,|\rho|=0.042)$ and Dothideomycetes also negatively correlated with iron concentrations $(\rho=-0.88,|\rho|=0.019)$. The summer AMD Eukaryote microbial community composition was dominated by Chlorophyceae and $L K M 11$, both positively correlated with drainage temperature $(\rho=0.92,|\rho|=0.008 \& 0.77$, $|\rho|=0.072)$ and negatively correlated with ice thickness $(\rho=-0.89,|\rho|=0.017 \&-0.93,|\rho|=0.008)$ and $\mathrm{pH}(\rho=0 .-92,|\rho|=0.008 \&-0.77,|\rho|=0.072)$ (RDA triplot, Figure 8B). The summer Archaeal community consisted of only two classes, Thermogymnomonas and Ferroplasma, and the abundance of both were only found to positively correlate with water temperature $(\rho=0.85,|\rho|=$ 0.035 and $0.89,|\rho|=0.0019$ respectively; due to the simplicity of the Archaeal community the Archaeal RDA analyses are not shown).

\section{Discussion}

\section{Copper Cliff AMD Community Profile Overview}

This study characterized the Bacterial, Eukaryote, and Archaeal microbial communities from an acid mine drainage site in Copper Cliff Ontario, across three winter and three summer dates. The community structure differed most substantially between the seasonal dates, indicating a distinct winter and summer community, with some variability also present within each of the two seasons. Across all the samples, Bacteria were the most diverse, followed by 
Eukaryotes and Archaea as the least diverse, including S1 and S2 samples points at which no Archaea were detected. Bacterial and Archaeal community diversity was highest in the winter months, while Eukaryote community diversity was relatively similar across the seasons. Across the three groups, the winter community is dominated by a few Bacterial and some Archaeal chemolithotrophs, with a less abundant population of Eukaryotic algae and fungal species. In the summer, the Bacterial heterotrophs were dominant with a notable absence of Archaea, and a community of Eukaryotes distinct from that observed during the winter. Our examination of AMD chemistry identified both expected and unexpected trends. Overall, the AMD metal and mineral concentrations were steady throughout both the winter and summer, although there was a sharp drop in sulfate, sulfur, copper, iron, nickel and a host of other metals by the end of winter (W3). This drop likely reflects the restriction by a solid ice cap of any new mineral rich seepage into the AMD basin during the winter. Given the harsh winter conditions in Northern Ontario (e.g. below freezing temperatures, heavy ice formation, lack of nutrient influx), we had expected total microbial diversity to be lower during the winter than summer. The unexpected higher diversity of winter Eukaryotic microbes may reflect a decreased dominance (near absence) of the bacterial heterotroph Acidiphilium. The winter Eukaryotes may take up the role of heterotroph, allowing Acidithiobacillus spp. to dominate the winter, with relative increases in other species (many unclassified) able tolerate colder temperatures, moderately higher $\mathrm{pH}$, and able to scavenge available nutrients. Even as iron and sulfur decrease, chemolithotrophs like Acidithiobacillus spp. remain active and continue to drive AMD and decreasing $\mathrm{pH}$ over the winter. Interestingly, the heterotrophs dominate during active summer AMD production and lowest $\mathrm{pH}$ levels indicating that even a smaller proportion of chemolithotrophs can maintain AMD production. 


\section{Bacterial AMD Community}

Acidithiobacillus spp. were dominant AMD community members during both summer and winter seasons and the most abundant identifiable iron oxidizer. Globally, Fe-S oxidizing species in the Acidithiobacillus and Leptospirillum genera have been identified as major contributors to the production of AMD (Kuang et al. 2013; Schrenk et al. 1998). However, their relative abundance and roles appear to be both site and environment specific (Mendez-Garcia et al. 2015), consistent with our observation that this site is dominated instead by Acidithiobacillus spp. with Leptospirillum spp. present, but rare.

While the overall dominance of Acidithiobacillus at this site is consistent with the previously described role of this group, the lower relative abundance during the summer, at least at S1 and $\mathrm{S} 2$, was surprising given the relatively mild environmental conditions. Strains of $A t$. ferrooxidans are known to be mesophilic with optimum growth temperatures of $\sim 26{ }^{\circ} \mathrm{C}$ (Baker and Banfield 2003), although species of Acidithiobacillus thrive under a variety of strainspecific, optimal growth temperatures (Kupka et al. 2007; Mykytczuk et al. 2010). At. ferrivorans, a psychrotolerant species (Liljeqvist et al. 2011) composed between 3-33\% of Acidithiobacillus sequence abundance throughout the winter at this site (higher averages in W1 and W2), clearly highlighting the role of cold-adapted iron/sulfur metabolism driving Fe-S oxidation during the winter. Several rare genera (OTU's $<1 \%$ ) found during winter, including Ignavibacterium, Sulfuricella, and Sulfuritalea, are sulfur oxidizing facultative anaerobes (Liu et al. 2012; Kojima and Fukui 2010; Kojima and Fukui 2011). The winter presence, and summer absence, of these organisms suggest that sub-optimal winter conditions for otherwise dominating iron oxidizing species such as At. ferrooxidans and L. ferrooxidans, may allow rare species, specifically sulfur oxidizing species, to be present in increased abundance and contribute to the 
depletion of the sulfur pool (sulfur and sulfate) over the winter. Consistent with this idea, other studies have also found cold-adapted, sulfur oxidizing communities that drive AMD during colder seasons at other sites (Elberling 2005; Kojima and Fukui 2010). Our results agree with similar findings that a cold-adapted community comprised of Acidithiobacillus sp., Acidobacteria and Gallionellaceae-like species, drive low temperature AMD processes in the Kristineberg mine in Sweden (Liljeqvist et al. 2015), however we found dominant members within the winter community that were not found at that site. Thus it is evident that a cold-active AMD community harbours different bacterial members, many of which do not yet have clearly defined metabolic roles in the environment, and even less understood are the Archaeal and Eukaryote members that help shape the low temperature AMD community.

The chemolithotrophs increased in abundance over the summer as the synergistic role of heterotrophs, like Acidiphilium, was evident in facilitating higher growth of both groups, as previously described (Baker and Banfield, 2003; Harrison 1984). Given this synergy, it is interesting that although Acidiphilium spp. are essentially absent at this site during the winter, Acidithiobacillus is able to remain abundant. This unexpected pattern suggests positive interactions between At. ferrivorans and cold-tolerant heterotrophs, possibly Herminiimonas, Flavobacteria, and/or eukaryotic heterotrophs, that keep organic carbon limited to allow continued Fe/S oxidation by At. ferrivorans.

\section{Eukaryote AMD Community}

The Eukaryotic AMD microbial communities have generally received less attention than either Bacterial or Archaeal AMD communities, but previous studies, including results reported here, suggest that this group makes up a substantial portion of the microbial community. 
Eukaryotic fungi and algae have been described in other AMD systems, but their effect on the AMD environment, and their response to changes in the environment, are still not well understood (Das et al. 2009). Globally, culture-independent techniques indicate that the relative abundance of Eukaryotes varies from site to site with some AMD sites, such as the Rio Tinto in Spain, being largely composed of Eukaryotes (Amaral Zettler et al. 2002; Amaral Zettler 2012) while other sites have relatively low Eukaryote diversity (Baker et al. 2009).

The winter Eukaryotic microbial community at our site is primarily comprised of algal and fungal species that shift in relative abundance throughout the season. The algal and fungal sequences largely belong to the two classes, Chrysophyceae and Microbotryomycetes respectively. Chrysophyceae, a large class of freshwater algae (Stoeck et al. 2009), represents over $85 \%$ of W1 and W2 Eukaryotes, and is also a dominant member of the AMD algal community at other AMD sites (Amaral Zettler et al. 2002). In the W3 sample, we saw a large community shift from algal to fungal species dominated by Microbotryomycetes, a subdivision of Basidiomycota which have also been previously isolated from mining contaminated, low $\mathrm{pH}$, environments (Jasrotia et al. 2014). Typically, algal growth is associated with warmer summer temperatures; however, high potential for algal winter growth has been demonstrated in and about sea ice at low temperatures, where algal species are found to proliferate (Schultz 2013). Algae have been proposed to positively affect the growth of both iron and sulfur reducing Bacterial heterotrophs by increasing the amount of dissolved organic carbon (Das et al. 2009; Nancucheo and Johnson 2012). Such a relationship could potentially support the winter AMD heterotroph community at this site.

During the summer, the Eukaryote community was initially composed largely of Chrysophyceae algal species, but the abundance of this group decreased through the summer as 
the abundance of the novel LKM 11 clade increased (to almost $65 \%$ at S3). The LKM 11 clade is a recently identified basal group of fungi, initially comprised solely of Rozellida; a group of endoparasites with algal and fungal hosts (Lara et al. 2010). Some species of parasitic fungi are host specific and are known to affect community composition (Gleason et al. 2012); such an effect could explain the drop in Chrysophyceae abundance through summer observed in this study.

Similarly, previous AMD studies at this and other locations have found Legionella species that were speculated to be endosymbionts within eukaryotic hosts (Auld et al, 2013; Hao et al. 2010). Legionella are typically not acid tolerant and, as such, are unlikely community members at AMD sites, but made up 8\% of Bacterial microbes in W3 (and more than 10\% of the community in our earlier study of this site; Auld et al 2013). In other systems, Legionella live endosymbiotically inside the vacuoles of amoeboid protists (Molmeret et al. 2005), a similar endosymbiotic relationship with an acidophilic ameboid protist would explain their presence in AMD sites (Hao et al. 2010). Our results include members of the phylum Cercozoa, a group that includes many amoeboids (Bass and Cavalier-Smith 2004) including Hartmannella amoeboids, found here during the winter, which have been implicated as hosts of endosymbiont Legionella spp. (Molmeret et al. 2005). Decreased temperatures during the winter can cause amoebae to differentiate into cysts (Ohno et al. 2008), within which Legionella have been found to survive (Kilvington and Price 1990), possibly explaining the survival of Legionella in AMD throughout the year when both Legionella and amoeboid sequences decrease or are absent.

\section{Archaeal AMD Community}


At this site, Archaea were a less abundant and diverse portion of the AMD microbial community than either bacteria or eukaryotes. The Archaeal community included genera of acidophilic heterotrophs, iron oxidizing species, and a notably high percentage of unclassified sequences. While relatively little is known about Archaea in AMD, we were able to identify Thermoplasmatales, including members from the Ferroplasma and Thermogymnomonas genera. This order includes acidophilic obligate, or facultative, aerobes and new genera are being uncovered from similar acid mine sites (Yelton et al. 2013). Recent metagenomic analyses of Thermoplasmatales suggest wide variation across members of this group in mobility, oxygen tolerance/growth, and metabolism, including iron and carbon monoxide oxidation and methylotrophy (Yelton et al. 2013). Ferroplasma spp. are capable of oxidizing both organic matter and ferrous iron (Huang et al. 2011; Edwards et al. 2000; Dopson et al. 2004) suggesting that they could play a role in the generation of AMD. Overall, Archaea appeared to be a transient, but potentially integral, component of the microbial AMD community, but their specific roles are still unknown.

\section{Conclusion}

Further insight into the microbial community diversity of AMD environments, and the seasonal changes and interactions between community members, is a valuable step toward better understanding AMD production and future control and remediation of such sites. Our results indicate that the AMD microbial community in all three domains varies seasonally, with distinct microbial communities driving nutrient and carbon cycling, and AMD production, in the winter and summer. Importantly, our findings provide baseline data needed when planning for management and treatment strategies. The abundance and diversity of winter-active chemolithotrophic bacteria strongly suggests that this is an active winter community whose 
ecology should not to be overlooked in our understanding of AMD production and our strategies for remediation. To counter these cold-active oxidative microbes, for example, winter addition of carbon substrates and inoculation of psychrophilic SRBs could promote reductive processes under the winter ice cover. Further, our results indicate that the Eukaryote microbial community, including algal and fungal species, likely plays a substantial role in carbon cycling within the AMD community. In total, the seasonal community turnover and shifts in diversity found at the Copper Cliff AMD site suggest a complex and dynamic seasonal microbial community and AMD production that needs to be considered when planning management solutions.

\section{Acknowledgments:}

We thank Mehrdad Hajibabaei for constructive comments on an earlier version of this manuscript and Glen Watson and Vale Ltd. for support and access to the tailings pond on Vale property. This work was supported by Natural Sciences and Engineering Research Council (NSERC) Discovery Grants to TJSM and NCSM and a Canada Research Chair to TJSM.

\section{Conflict of Interest:}

The authors declare that they have no conflict of interest.

\section{Bibliography}

Altschul, Stephen F., Warren Gish, Webb Miller, Eugene W. Myers, and David J. Lipman. 1990. "Basic local alignment search tool." Journal of molecular biology 215.3: 403-410.

Amaral-Zettler, L. A., F. Gómez, E. Zettler, B. G. Keenan, R. Amils, and M. L. Sogin. 2002. "Microbiology: Eukaryotic Diversity in Spain's River of Fire." Nature 417: 137.

Amaral-Zettler, Linda A. 2012. "Eukaryotic diversity at pH extremes." Frontiers in microbiology 3,441 .

Andreotti, R., A. A. León, S. E. Dowd, F. D. Guerrero, K. G. Bendele, and G. A. Scoles. 2011. "Assessment of Bacterial Diversity in the Cattle Tick Rhipicephalus (Boophilus) Microplus through Tag-encoded Pyrosequencing." BMC Microbiology 11.1: 6.

Auld, R. R., M. Myre, N. C. Mykytczuk, L. G. Leduc, and T. J. Merritt. 2013. "Characterization of the Microbial Acid Mine Drainage Microbial Community Using Culturing and Direct Sequencing Techniques." Journal of Microbiological Methods 93.2: 108-15. 
Baker, B. J., G. W. Tyson, L. Goosherst, and J. F. Banfield. 2009. "Insights into the Diversity of Eukaryotes in Acid Mine Drainage Biofilm Communities." Applied and Environmental Microbiology 75.7: 2192-199.

Baker, B. J., G. W. Tyson, R. I. Webb, J. Flanagan, P. Hugenholtz, E. E. Allen, and J. F. Banfield. 2006. "Lineages of Acidophilic Archaea Revealed by Community Genomic Analysis." Science 314.5807: 1933-935.

Baker, B. J., and J. F. Banfield. 2003. "Microbial Communities in Acid Mine Drainage." FEMS Microbiology Ecology 44: 139-52.

Bass, D., and T. Cavalier-Smith. 2004. "Phylum-specific Environmental DNA Analysis Reveals Remarkably High Global Biodiversity of Cercozoa (Protozoa)." International Journal Of Systematic And Evolutionary Microbiology 54.6: 2393-404.

Bond, P. L., G. K. Druschel, and J. F. Banfield. 2000. "Comparison of Acid Mine Drainage Microbial Communities in Physically and Geochemically Distinct Ecosystems." Applied and Environmental Microbiology 66.11: 4962-971.

Buckeridge, K. M., S. Banerjee, S. D. Siciliano, and P. Grogan. 2013. "The Seasonal Pattern of Soil Microbial Community Structure in Mesic Low Arctic Tundra." Soil Biology and Biochemistry 65: 338-47.

Caporaso, J. G., J. Kuczynski, J. Stombaugh, K. Bittinger, F. D. Bushman, E. K. Costello, N. Fierer, A. G. Peña, J. K. Goodrich, J. I. Gordon, G. A. Huttley, S. T. Kelley, D. Knights, J. E. Koenig, R. E. Ley, C. A. Lozupone, D. McDonald, B. D. Muegge, M. Pirrung, J. Reeder, J. R. Sevinsky, P. J. Turnbaugh, W. A. Walters, J. Widmann, T. Yatsunenko, J. Zaneveld, and R. Knight. 2010. "QIIME Allows Analysis of High-throughput Community Sequencing Data." Nature Methods 7.5: 335-36.

Caporaso, J. G., C. L. Lauber, W. A. Walters, D. Berg-Lyons, C. A. Lozupone, P. J. Turnbaugh, N. Fierer, and R. Knight. 2011. "Colloquium Paper: Global Patterns of 16S RRNA Diversity at a Depth of Millions of Sequences per Sample." Proceedings of the National Academy of Sciences 108: 4516-522.

Chao, A. 1987. "Estimating the Population Size for Capture-recapture Data with Unequal Catchability." Biometrics 43: 783-91.

Das, B. K., A. Roy, M. Koschorreck, S. M. Mandal, K. Wendt-Potthoff, and J. Bhattacharya. 2009. "Occurrence and Role of Algae and Fungi in Acid Mine Drainage Environment with Special Reference to Metals and Sulfate Immobilization." Water Research 43.4: 883-94.

Dopson, M., C. Baker-Austin, A. Hind, J. P. Bowman, and P. L. Bond. 2004. "Characterization of Ferroplasma Isolates and Ferroplasma Acidarmanus Sp. Nov., Extreme Acidophiles from 
Acid Mine Drainage and Industrial Bioleaching Environments." Applied and Environmental Microbiology 70.4: 2079-088.

Dowd, S. E., Callaway, T. R., Wolcott, R. D., Sun, Y., McKeehan, T., Hagevoort, R. G., \& Edrington, T. S. 2008. "Evaluation of the bacterial diversity in the feces of cattle using 16S rDNA bacterial tag-encoded FLX amplicon pyrosequencing (bTEFAP)." BMC microbiology, 8 . 1: 125 .

Edwards, K. J., P. L. Bond, T. M. Gihring, and J. F. Banfield. 2000. "An Archaeal IronOxidizing Extreme Acidophile Important in Acid Mine Drainage." Science 287.5459: 1796-799.

Edwards, K. J., T. M. Gihring, and J. F. Banfield. 1999. "Seasonal Variations in Microbial Populations and Environmental Conditions in an Extreme Acid Mine Drainage Environment." Applied and Environmental Microbiology 65.8: 3627-632.

Egiebor, N. O., and B. Oni. 2007. "Acid Rock Drainage Formation and Treatment: A Review." Asia-Pacific Journal of Chemical Engineering 2.1: 47-62.

Elberling, B. 2005. "Temperature and Oxygen Control on Pyrite Oxidation in Frozen Mine Tailings." Cold Regions Science and Technology 41.2: 121-33.

Gilbert, J. A., J. A. Steele, J. Gregory Caporaso, L. Steinbrück, J. Reeder, B. Temperton, S. Huse, A. C. Mchardy, R. Knight, I. Joint, P. Somerfield, J. A. Fuhrman, and D. Field. 2011. "Defining Seasonal Marine Microbial Community Dynamics." The ISME Journal 6.2: 298-308.

Gleason, F. H., L. T. Carney, O. Lilje, and S. L. Glockling. 2012. "Ecological Potentials of Species of Rozella (Cryptomycota)." Fungal Ecology 5.6: 651-56.

Hallberg, K. B., K. Coupland, S. Kimura, and D. B. Johnson. 2006. "Macroscopic Streamer Growths in Acidic, Metal-Rich Mine Waters in North Wales Consist of Novel and Remarkably Simple Bacterial Communities." Applied and Environmental Microbiology 72.3: 2022-030.

Hallberg, K. B. 2010. "New Perspectives in Acid Mine Drainage Microbiology." Hydrometallurgy 104: 448-53.

Haas, B. J., Gevers, D., Earl, A. M., Feldgarden, M., Ward, D. V., Giannoukos, G., Ciulla, D., Tabbaa, D., Highlander, S. K., Sodergren, E., Methe, B., DeSantis, T. Z., Petrosino, J. F., Knight, R., and Birren, B. W. 2011. Chimeric 16S rRNA sequence formation and detection in Sanger and 454-pyrosequenced PCR amplicons. Genome Research, 21.3: 494-504.

Hao, C., L. Wang, Y. Gao, L. Zhang, and H. Dong. 2010. "Microbial Diversity in Acid Mine Drainage of Xiang Mountain Sulfide Mine, Anhui Province, China." Extremophiles 14.5: 46574.

Harrison A. P. 1984. The acidophilic thiobacilli and other acidophilic bacteria that share their habitat. Annual Reviews in Microbiology, 38.1: 265-292. 
Huang, L., W. Zhou, K. Hallberg, C. Wan, J. Li, and W. Shu. 2011. "Spatial and Temporal Analysis of the Microbial Community in the Tailings of a Pb-Zn Mine Generating Acidic Drainage." Applied and Environmental Microbiology 77.15: 5540-544.

Jasrotia, P., Green, S. J., Canion, A., Overholt, W. A., Prakash, O., Wafula, D., Hubbard, D., Watson, D. B., Schadt, C. W., Brooks, S. C., and Kostka, J. E. 2014. Watershed-Scale Fungal Community Characterization along a $\mathrm{pH}$ Gradient in a Subsurface Environment Cocontaminated with Uranium and Nitrate. Applied and Environmental Microbiology, 80(6): 1810-1820.

Kay, C. M., Rowe, O. F., Rocchetti, L., Coupland, K., Hallberg, K. B., \& Johnson, D. B. 2013. Evolution of microbial "streamer" growths in an acidic, metal-contaminated stream draining an abandoned underground copper mine. Life, 3.1: 189-210.

Kilvington, S., and J. Price. 1990. "Survival of Legionella Pneumophila within Cysts of Acanthamoeba Polyphaga following Chlorine Exposure." Journal of Applied Microbiology 68.5: 519-25.

Kojima, H., and M. Fukui. 2011. "Sulfuritalea Hydrogenivorans Gen. Nov., Sp. Nov., a Facultative Autotroph Isolated from a Freshwater Lake." Int J Syst Evol Microbiol 61.7: 1651655.

Kojima, H., and M. Fukui. 2010. "Sulfuricella Denitrificans Gen. Nov., Sp. Nov., a Sulfuroxidizing Autotroph Isolated from a Freshwater Lake." Int J Syst Evol Microbiol 60.12: 2862866.

Kuang, J., L. Huang, L. Chen, Z. Hua, S. Li, M. Hu, J. Li, and W. Shu. 2013. "Contemporary Environmental Variation Determines Microbial Diversity Patterns in Acid Mine Drainage." The ISME Journal, 7.5: 1038-050.

Kupka, D., Rzhepishevska, O. I., Dopson, M., Lindström, E. B., Karnachuk, O. V., \& Tuovinen, O. H. 2007. "Bacterial oxidation of ferrous iron at low temperatures." Biotechnology and Bioengineering 97.6: 1470-1478.

Lara, E., Moreira, D., \& López-García, P. 2010. The Environmental Clade LKM11 and Rozella Form the Deepest Branching Clade of Fungi. Protist, 161(1): 116-121.

Leduc, D., L. G. Leduc, and G. D. Ferroni. 2002. "Quantification of Bacterial Populations Indigenous to Acidic Drainage Streams." Water, Air, and Soil Pollution 135.1-4: 1-21.

Liljeqvist, M., Ossandon, F.J., Gonzalez, C., Rajan, S., Stell, A., Valdes, J., Holmes, D.S. and Dopson, M. 2015. "Metagenomic analysis reveals adaptations to a cold-adapted lifestyle in a low-temperature acid mine drainage stream." FEMS microbiology ecology, 91, 4: fiv011. 
Liljeqvist, M., J. Valdes, D. S. Holmes, and M. Dopson. 2011. "Draft Genome of the Psychrotolerant Acidophile Acidithiobacillus Ferrivorans SS3." Journal of Bacteriology 193.16: 4304-305.

Liu, Z., N. U. Frigaard, K. Vog1, T. Iino, M. Ohkuma, J. Overmann, and D. A. Bryant. 2012. "Complete Genome of Ignavibacterium Album, a Metabolically Versatile, Flagellated, Facultative Anaerobe from the Phylum Chlorobi." Frontiers in Microbiology 3.185: 1-15.

Lozupone, C., and R. Knight. 2005. "UniFrac: A New Phylogenetic Method for Comparing Microbial Communities." Applied and Environmental Microbiology 71.12: 8228-235.

McGinness, S., \& Johnson, D. B. 1993. Seasonal variations in the microbiology and chemistry if an acid mine drainage stream. Science of the total environment, 132.1: 27-41.

Medlin, L., H. J. Elwood, S. Stickel, and M. L. Sogin. 1988. "The Characterization of Enzymatically Amplified Eukaryotic 16S-like RRNA-coding Regions." Gene 71.2: 491-99.

MEND, 1994. Canada Wide Survey of Acid Mine Drainage Characteristics. Wilson, L.J., Mining Environment Neutral Drainage Program. CANMET, Canada Centre for Mineral and Energy Technology.

Méndez-García, C., Peláez, A. I., Mesa, V., Sánchez, J., Golyshina, O. V., \& Ferrer, M. 2015. Microbial diversity and metabolic networks in acid mine drainage habitats. Frontiers in Microbiology, 6: 475.

Molmeret, M., M. Horn, M. Wagner, M. Santic, and Y. Abu Kwaik. 2005. "Amoebae as Training Grounds for Intracellular Bacterial Pathogens." Applied and Environmental Microbiology 71.1: 20-28.

Mykytczuk, N. C.S, J. T. Trevors, S. M. Twine, G. D. Ferroni, and L. G. Leduc. 2010. "Membrane Fluidity and Fatty Acid Comparisons in Psychrotrophic and Mesophilic Strains of Acidithiobacillus Ferrooxidans under Cold Growth Temperatures." Archives of Microbiology 192.12: 1005-018.

Nancucheo, I., and D. B. Johnson. 2012. "Acidophilic Algae Isolated from Mine-impacted Environments and Their Roles in Sustaining Heterotrophic Acidophiles." Frontiers in Microbiology 3.325: 1-8.

Nocker, A., C. Cheung, and A. K. Camper. 2006. "Comparison of Propidium Monoazide with Ethidium Monoazide for Differentiation of Live vs. Dead Bacteria by Selective Removal of DNA from Dead Cells." Journal of Microbiological Methods 67.2: 310-20.

Ohno, A., N. Kato, R. Sakamoto, S. Kimura, and K. Yamaguchi. 2008. "TemperatureDependent Parasitic Relationship between Legionella Pneumophila and a Free-Living Amoeba (Acanthamoeba Castellanii)." Applied and Environmental Microbiology 74.14: 4585-588. 
Paster, B. J., W. A. Falkler, C. O. Enwonwu, E. O. Idigbe, K. O. Savage, V. A. Levanos, M. A. Tamer, R. L. Ericson, C. N. Lau, and F. E. Dewhirst. 2002. "Prevalent Bacterial Species and Novel Phylotypes in Advanced Noma Lesions." Journal of Clinical Microbiology 40.6: 2187191.

Quast, C., E. Pruesse, P. Yilmaz, J. Gerken, T. Schweer, P. Yarza, J. Peplies, and F. O. Glockner. 2013. "The SILVA Ribosomal RNA Gene Database Project: Improved Data Processing and Web-based Tools." Nucleic Acids Research 41,D1: 590-96.

Remonsellez, F. Galleguillos, M. Moreno-Paz, V. Parro, M. Acosta, C. Demergass. 2009. Dynamic of active microorganisms inhabiting a bioleaching industrial heap of low-grade copper sulfide ore monitored by real-time PCR and oligonucleotide prokaryotic acidophile microarray Microb. Biotechnol, 2.6: 613-624

Schrenk, M. O., K. J. Edwards, R. M. Goodman, R. J. Hamers, and J. F. Banfield. 1998. "Distribution of Thiobacillus Ferrooxidans and Leptospirillum Ferrooxidans: Implications for Generation of Acid Mine Drainage." Science 279.5356: 1519-522.

Shannon, C. E., and W. Weaver. 1949. The Mathematical Theory of Communication. Urbana: University of Illinois.

Stoeck, T., A. Behnke, R. Christen, L. Amaral-Zettler, M. J. Rodriguez-Mora, A. 2009. Chistoserdov, W. Orsi, and V. P. Edgcomb. "Massively Parallel Tag Sequencing Reveals the Complexity of Anaerobic Marine Protistan Communities." BMC Biology 7.1: 72.

Tamura, K., D. Peterson, N. Peterson, G. Stecher, M. Nei, and S. Kumar. 2011. "MEGA5: Molecular Evolutionary Genetics Analysis Using Maximum Likelihood, Evolutionary Distance, and Maximum Parsimony Methods." Molecular Biology and Evolution 28.10: 2731-739.

Tan, G. L., W. S. Shu, W. H. Zhou, X. L. Li, C. Y. Lan, and L. N. Huang. 2009. "Seasonal and Spatial Variations in Microbial Community Structure and Diversity in the Acid Stream Draining across an Ongoing Surface Mining Site." FEMS Microbiology Ecology 70.2: 121-29.

Tan, G., W. Shu, K. B. Hallberg, F. Li, C. Lan, and L. Huang. 2007. "Cultivation-dependent and Cultivation-independent Characterization of the Microbial Community in Acid Mine Drainage Associated with Acidic $\mathrm{Pb} / \mathrm{Zn}$ Mine Tailings at Lechang, Guangdong, China." FEMS Microbiology Ecology 59.1: 118-26.

Volant, A., Bruneel, O., Desoeuvre, A., Héry, M., Casiot, C., Bru, N., Delpoux, S., Fahy, A., Javerliat, F., Bouchez, O. and Duran, R. 2014. Diversity and spatiotemporal dynamics of bacterial communities: physicochemical and other drivers along an acid mine drainage. FEMS microbiology ecology, 90.1: 247-263.

Wang, Q., G. M. Garrity, J. M. Tiedje, and J. R. Cole. 2007. "Naive Bayesian Classifier for Rapid Assignment of RRNA Sequences into the New Bacterial Taxonomy." Applied and Environmental Microbiology 73.16: 5261-267. 
Weekers, P. H. H, R. J. Gast, P. A. Fuerst, and T. J. Byers. 1994. "Sequence Variations in Smallsubunit Ribosomal-RNAs of Hartmannella Vermiformis and Their Phylogenetic Implications." Molecular Biology and Evolution 11: 684-90.

Yelton, A. P., L. R. Comolli, N. B. Justice, C. Castelle, V. J. Denef, B. C. Thomas, and J. F. Banfield. 2013. "Comparative Genomics in Acid Mine Drainage Biofilm Communities Reveals Metabolic and Structural Differentiation of Co-occurring Archaea." BMC Genomics 14.1: 485.

\section{Figure Legends}

Fig 1

Acid mine drainage (AMD) sampling site, Copper Cliff, Ontario Canada; three pond locations were used for drainage sampling labelled A, B, C. Image Source Google Earth (June 2015).

\section{Fig 2}

Water conditions and metal concentrations for each of the six sampling dates. For each characteristic, trend-lines and axes share a color (i.e. temperature is indicated with fuchsia circles and lines and in fuchsia numbers on the $\mathrm{Y}$-axis).

\section{Fig 3}

Bacterial (A), Archaeal (B), and Eukaryote (C) weighted Unifrac PCoA plots depicting seasonal community similarities. Triplicate sample communities are represented; W1 (Blue), W2 (Light blue), and W3 (Purple) represents the first, second, and third winter sampling dates, and S1 (Red), S2 (Orange), and S3 (Yellow), the first, second, and third summer dates respectively.

\section{Fig 4}

Seasonal Bacterial AMD community profile depicted by relative percent sequence abundance at class (A) and genus (B) levels. Bars W1 - W3 and S1 - S3 represent the community profiles for the first to third winter and summer sampling dates, each date comprising of triplicate sequencing analyses.

\section{Fig 5}

Maximum likelihood phylogeny of dominant ( $>1 \%)$ Bacterial OTU's and their closest BLAST hits. Red and black symbols indicate winter and summer sequencing OTU's respectively, and bracketed values represent OTU abundances along condensed monophyletic groups.

\section{Fig 6}

Seasonal Archaeal AMD community profile depicted by relative percent sequence abundance at class (A) and genus (B) levels. Archaea were essentially absent (no sequencing data was obtained, see methods) for S1 and S2.

\section{Fig 7}

Seasonal Eukaryote AMD class community profile depicted by relative percent sequence abundance.

\section{Fig 8}


Bacterial (A) and Eukaryote (B) redundancy analysis (RDA) triplots, illustrating the relationship between the bacterial AMD community, species, and AMD chemical and physical properties. 